\title{
Synaptic dysfunction, memory deficits and hippocampal atrophy due to ablation of mitochondrial fission in adult forebrain neurons
}

\author{
B Oettinghaus ${ }^{1}$, JM Schulz ${ }^{2}$, LM Restelli ${ }^{1}$, M Licci $^{1,3}$, C Savoia $^{4}$, A Schmidt ${ }^{5}, K$ Schmitt ${ }^{6}$, A Grimm ${ }^{6}$, L Morè ${ }^{7}$, J Hench $^{1}$, M Tolnay ${ }^{1}$, \\ A Eckert ${ }^{6}$, P D'Adamo $^{7}$, P Franken ${ }^{8}, \mathrm{~N}$ Ishihara ${ }^{9}, \mathrm{~K}$ Mihara $^{9,10}$, J Bischofberger ${ }^{2}$, L Scorrano ${ }^{*, 4,11}$ and S Frank ${ }^{\star, 1}$
}

\begin{abstract}
Well-balanced mitochondrial fission and fusion processes are essential for nervous system development. Loss of function of the main mitochondrial fission mediator, dynamin-related protein 1 (Drp1), is lethal early during embryonic development or around birth, but the role of mitochondrial fission in adult neurons remains unclear. Here we show that inducible Drp1 ablation in neurons of the adult mouse forebrain results in progressive, neuronal subtype-specific alterations of mitochondrial morphology in the hippocampus that are marginally responsive to antioxidant treatment. Furthermore, DRP1 loss affects synaptic transmission and memory function. Although these changes culminate in hippocampal atrophy, they are not sufficient to cause neuronal cell death within 10 weeks of genetic Drp1 ablation. Collectively, our in vivo observations clarify the role of mitochondrial fission in neurons, demonstrating that Drp1 ablation in adult forebrain neurons compromises critical neuronal functions without causing overt neurodegeneration.
\end{abstract}

Cell Death and Differentiation (2016) 23, 18-28; doi:10.1038/cdd.2015.39; published online 24 April 2015

In addition to their crucial importance in energy conversion, mitochondria serve many other housekeeping functions, including calcium buffering, amino-acid and steroid biosynthesis as well as fatty acids beta-oxidation and regulation of cell death. During the past decade, it has become increasingly clear that processes regulating mitochondrial morphology and ultrastructure are influenced by specific cellular requirements upon which mitochondria, in a precisely regulated manner, undergo fusion and division events. ${ }^{1}$ Maintaining this balance is especially important for highly energyconsuming, polarized cells such as neurons, where single organellar units sprouting from the mitochondrial network are transported along the cytoskeleton into dendrites and spines to meet local energy requirements. ${ }^{2}$ In addition, elaborate quality-control mechanisms also rely on mitochondrial dynamics: whereas defective organelles are sequestered by fission, enabling their removal from the mitochondrial network, ${ }^{3,4}$ fusion supports qualitative homogeneity of the syncytium through complementation. ${ }^{5}$

Mitochondrial fusion and fission are mediated by large GTPases of the dynamin superfamily. ${ }^{6}$ The outer mitochondrial membrane mitofusins 1 (MFN1) and 2 (MFN2) tether mitochondrial membranes by homodimer or heterodimer formation, ${ }^{7}$ thereby initiating fusion of the organelles, a process that also involves the inner mitochondrial membraneassociated GTPase Optic Atrophy $1 .^{8}$ In addition, MFN2 also mediates contacts between mitochondria and endoplasmic reticulum. ${ }^{9}$ The only known mammalian mitochondrial fission protein, Dynamin-Related Protein 1 (Drp1), translocates upon dephosphorylation by calcineurin ${ }^{10}$ to fission sites where it binds to mitochondrial fission factor. ${ }^{11}$ Drp 1 translocation is preceded by ER membranes wrapping around mitochondria to constrict the organelles, ${ }^{12}$ thereby facilitating the formation of multimeric Drp1 complexes that, upon GTP hydrolysis, further tighten to complete the process of mitochondrial fission. ${ }^{13}$

Genetic evidence in mice and humans indicates that mitochondrial dynamics are crucially important in neurons: in humans, a sporadic dominant-negative DRP1 mutation caused a lethal syndromic defect with abnormal brain development; ${ }^{14}$ similarly, constitutive Drp1 knockout in the mouse brain leads to lethal neurodevelopmental defects. ${ }^{15,16}$ Although the

${ }^{1}$ Division of Neuropathology, Institute of Pathology, University Hospital Basel, Basel 4031, Switzerland; ${ }^{2}$ Division of Neurophysiology, Institute of Physiology, Department of Biomedicine, University of Basel, Basel 4056, Switzerland; ${ }^{3}$ Department of Neurosurgery, University Hospital Basel, Basel 4031, Switzerland; ${ }^{4}$ Department of Biology, University of Padua, Padua 35121, Italy; ${ }^{5}$ Proteomics Core Facility, Biozentrum, University of Basel, Basel 4056, Switzerland; ${ }^{6}$ Neurobiology Laboratory for Brain and Mental Health, University Psychiatric University Clinics Basel, University of Basel, Basel 4012, Switzerland; ${ }^{7}$ Dulbecco Telethon Institute IRCCS, San Raffaele Scientific Institute, Milan 20132, Italy; ${ }^{8}$ Faculty of Biology and Medicine, Center for Integrative Genomics, University of Lausanne, Lausanne 1015, Switzerland; ${ }^{9}$ Department of Protein Biochemistry, Institute of Life Science, Kurume University, Kurume 839-0864, Japan; ${ }^{10}$ Department of Molecular Biology, Graduate School of Medical Science, Kyushu University, Fukuoka 812-8582, Japan and ${ }^{11}$ Dulbecco Telethon Institute, Venetian Institute of Molecular Medicine, Padua 35129, Italy

${ }^{*}$ Corresponding author: L Scorrano, Department of Biology, University of Padua, Padua 35121, Italy; Tel: +39 0498276320; Fax: +39 0497923271; E-mail: luca.scorrano@unipd.it

or S Frank, Division of Neuropathology, Institute of Pathology, University Hospital Basel, Schoenbeinstrasse 40, Basel 4031, Switzerland. Tel: +41612652776 ; Fax: +41 61 2653194; E-mail: stephan.frank@usb.ch

Abbreviations: DG, dentate gyrus; fEPSP, field excitatory postsynaptic potential; H\&E, hematoxylin-eosin; i $\Delta \mathrm{b}$, inducibly ablated in the brain; p.t.i., posttamoxifen injection; TEM, transmission electron microscopy; TUNEL, TdT-mediated dUTP-biotin nick end labeling; ROS, reactive oxygen species

Received 24.10.14; revised 27.2.15; accepted 03.3.15; Edited by J-C Marine; published online 24.4.15 
crucial role of Drp1 during brain development is undisputed, studies on Drp1 function in postmitotic (adult) neurons are scarce; likewise, Drp1 ablation studies in primary cultures have so far failed to yield a conclusive picture. In vitro, Drp1 ablation is reported to lead to a super-elongated neuroprotective $^{17-24}$ or an aggregated mitochondrial phenotype associated with neurodegeneration. ${ }^{15,16,25-27}$ These discrepancies are probably due to different experimental conditions: neuronal health is indeed influenced by the onset and duration of Drp1 inhibition, which varies considerably among the cited reports, ${ }^{28}$ and different types of neuronal cultures studied display different sensitivity to Drp1 inhibition. In vivo, Drp1 ablation in Purkinje cells results in oxidative stress and neurodegeneration, ${ }^{29}$ demonstrating that Drp1 is essential for postmitotic neurons' health. In contrast, transient pharmacological Drp1 inhibition is neuroprotective in several mouse ischemia models, indicating that temporarily blocking mitochondrial fission holds therapeutic potential. ${ }^{30-32}$

To elucidate the consequences of blocked mitochondrial fission in the central nervous system in vivo, we bypassed the critical role of Drp1 during brain development by generating Drp1 ${ }^{f l x / f l x}$ mice ${ }^{15}$ expressing tamoxifen-inducible Cre recombinase under the control of the CaMKlla promoter. ${ }^{33}$ Upon induced Drp1 deletion in postmitotic adult mouse forebrain neurons, mice develop progressive, neuronal subtype-specific alterations in mitochondrial shape and distribution in the absence of overt neurodegeneration. In addition, respiratory capacity, ATP content, synaptic reserve pool vesicle recruitment as well as spatial working memory are impaired, demonstrating that severely dysregulated mitochondrial dynamics can compromise critical neuronal functions in vivo without causing neuronal cell death.

\section{Results}

Inducible Drp1 ablation in the adult mouse forebrain. In order to study the effect of mitochondrial fission ablation in adult neurons, we generated a model of inducible Drp1 ablation in the forebrain by crossing mice with loxP sites inserted in the Drp1 gene $\left(D r p 1^{f l x / f l x}\right)^{15}$ with mice expressing a gene for a tamoxifen-inducible Cre recombinase fusion protein under the control of the CaMKIla promoter (Figure 1a), ${ }^{33}$ limiting recombination primarily to the forebrain. Eight-weekold offspring of these crossings were injected with tamoxifen on 5 consecutive days (Drp1 inducibly deleted in the brain, $\left.D r p 1^{i \Delta b / i \Delta b}\right)$, resulting in maximum DRP1 depletion in the hippocampus within 14 days (Figure 1b). Immunohistochemical DRP1 staining in Drp $1^{i \Delta b / \Delta b}$ animals 4 weeks posttamoxifen injection (p.t.i.) confirmed that DRP1 was lacking specifically in hippocampal neurons (Figure 1c) and crosses of $D r p 1^{i \Delta b / \Delta b}$ mice with a reporter strain further confirmed the recombination following tamoxifen injection (Supplementary Figure S1A).

Drp1 ablation causes progressive changes in mitochondrial morphology. To characterize the consequences of inducible Drp1 ablation, we turned to an in vitro model of primary cortical neurons isolated from $\operatorname{Drp} 1^{f l x / f l x}$ mice where we could monitor by confocal microscopy the consequences of Cre recombinase-induced Drp1 ablation by co-transfecting it with a mitochondria-targeted yellow fluorescent protein (mtYFP). Starting at day 3 following in vitro recombination, mitochondria progressively clustered and aggregated in the soma. Fewer organelles were found within the neurite compartment, where mitochondria also appeared larger a

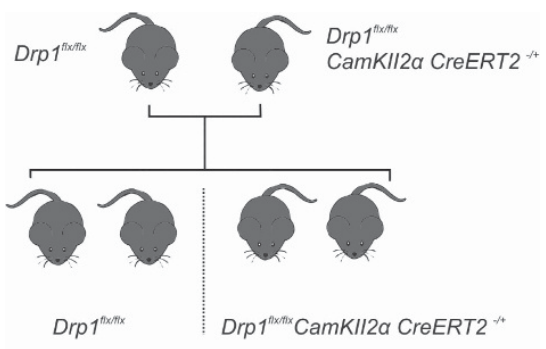

Tamoxifen injection at 8 weeks of age induces recombination of the Drp1 locus

b

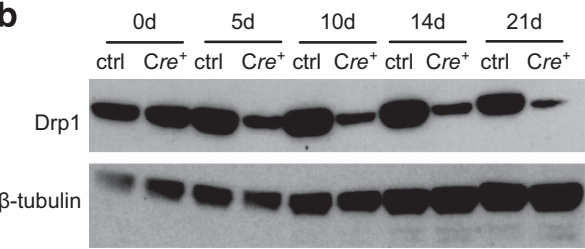

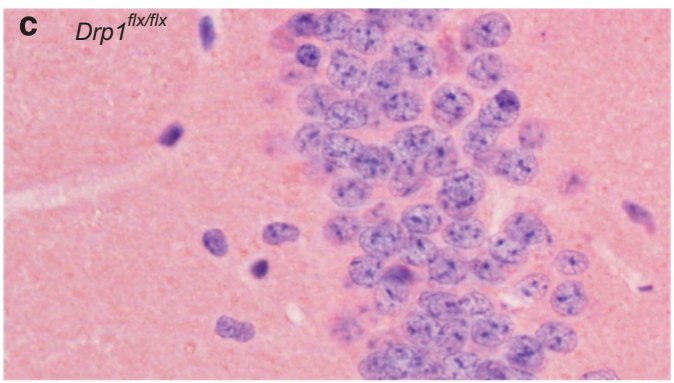

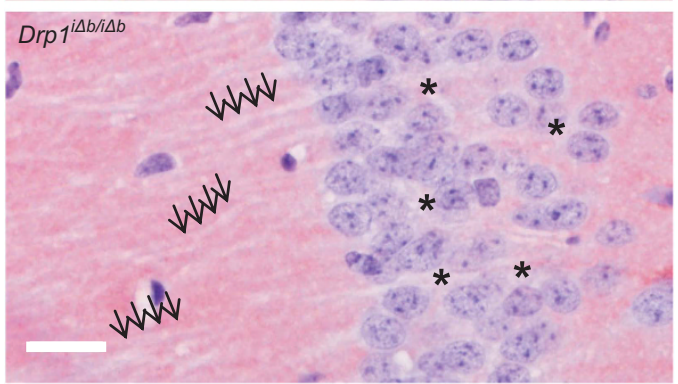

Figure 1 Drp1 ablation in postmitotic forebrain neurons. (a) Drp1 $1^{f \mid x f l x}$ and Drp $1^{f \mid x / f x}$ CaMKIl $\alpha$ CreERT2 ${ }^{-/+}$mice were crossed, resulting in a Mendelian distribution of alleles. At 8 weeks of age, animals were injected with tamoxifen for 5 consecutive days to induce recombination of the floxed Drp 1 locus. (b) Mice of the indicated genotype were killed at the indicated time points p.t.i. Hippocampal lysates were separated by sodium dodecyl sulfate-polyacrylamide gel electrophoresis and immunoblotted using the indicated antibodies. (c) Immmunohistochemical staining (NovaRED (Vectorlabs, Burlingame, CA, USA), counterstain hematoxylin) for Drp1 was performed on hippocampal CA1 formalinfixed, paraffin-embedded tissue sections of $D r p 1^{i \Delta b / \Delta b}$ mice 4 weeks p.t.i. Arrows indicate unstained hippocampal dendrites. Scale bar, $5 \mu \mathrm{m}$ 

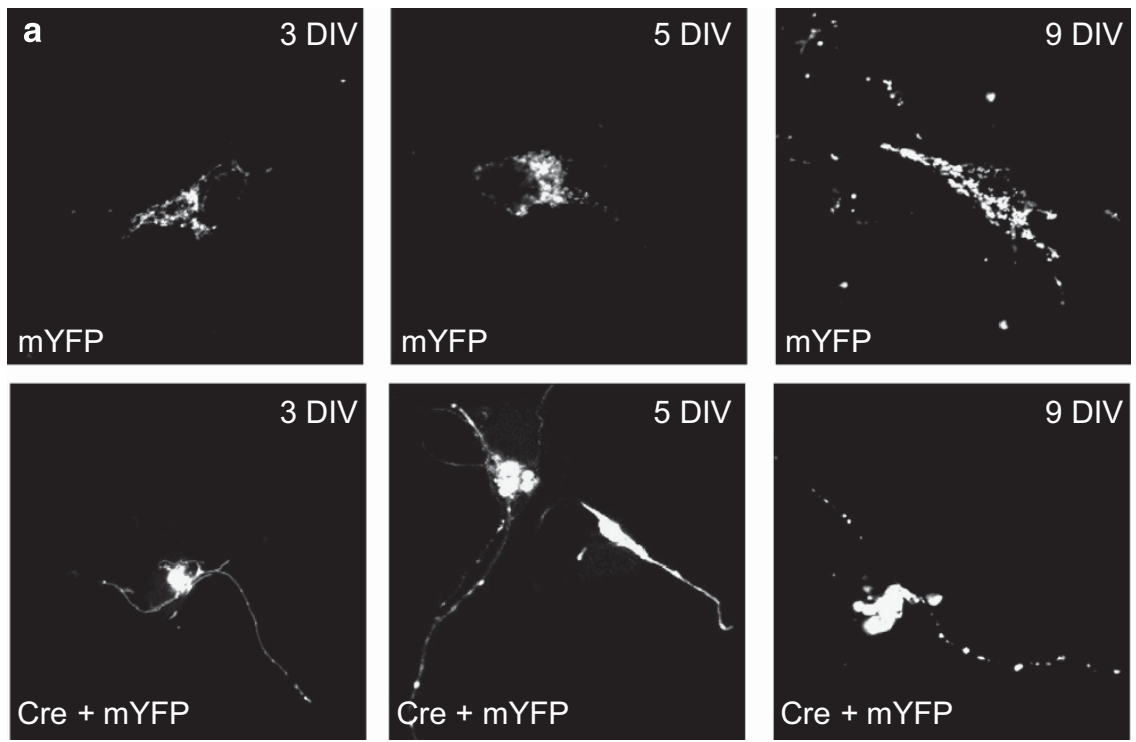

Figure 2 Mitochondrial morphology in primary neuronal cultures after Drp1 ablation. (a) Primary cortical neurons isolated from Drp1 ${ }^{f x f f i x}$ E17 embryos were coelectroporated with mtYFP- and Cre-expression constructs prior to seeding. Images were taken at the indicated time points of differentiation

in comparison to sham-transfected controls (Figure 2). Comforted by these results, we moved to adult hippocampal neurons in vivo to study mitochondrial morphology by immunohistochemistry. At day 10 after Drp1 ablation, enlarged spherical mitochondria started to aggregate in the perikarya of hippocampal neurons. Whereas 10 days p.t.i. enlarged mitochondria clustered in the perikarya of approximately only $5 \%$ of CA1 neurons, in the dentate gyrus (DG) and CA3 hippocampal neurons, mitochondrial morphology changes occurred earlier after Drp1 ablation. Conversely, 4 weeks p.t.i., neuronal mitochondrial morphology was heavily altered in all three neuronal subpopulations, mitochondria of CA3 pyramidal neurons appearing considerably less filamentous and more fragmented compared with CA1 and DG neurons (Figure 3). These findings demonstrate that blocking fission alters mitochondrial morphology and distribution in a neuronal subtype-specific manner.

Synaptic transmission is impaired in Drp1-deficient CA1 pyramidal neurons. We next addressed whether the observed mitochondrial morphological defects resulted in any functional consequence by behavioral and electrophysiological tests. Biocytin filling indicated that dendritic morphology of hippocampal neurons was not altered 4 weeks p.t.i. (Figure 4a). Accordingly, whole-cell patch-clamp recordings revealed that CA1 pyramidal Drp $1^{i \Delta b / \Delta b}$ neurons display normal resting membrane potential and action potential peak amplitudes as well as half-duration (Figures $4 b$ and e). When we explored excitatory synaptic transmission by performing field potential recordings in the hippocampal $\operatorname{Drp} 1^{i \Delta b / i \Delta b}$ CA1 region, we observed that stimulation of the Schaffer collaterals evoked field excitatory postsynaptic potentials (fEPSPs) with normal paired-pulse facilitation (143\%, $n=10 ; \Delta t=100 \mathrm{~ms}$ ) similar to those observed in control mice $(151 \%, n=6, P=0.52)$, indicating normal release probability. However, when synaptic transmission was challenged by application of 100 stimuli at either 10 or $100 \mathrm{~Hz}$, frequency-dependent fEPSP facilitation was significantly reduced in $D r p 1^{i \Delta b / \Delta b}$ brain slices, where synaptic transmission also broke down more rapidly (Figures 5a and c; Supplementary Figures S1D and E). In addition, when subjected to a spontaneous alternation task (a behavioral assay for hippocampus-dependent working memory), shortterm working memory was significantly impaired in $\operatorname{Drp} 1^{i \Delta b / \Delta b}$ mice compared with their Drp $1^{f \mid x / f l x}$ littermates (Figure $5 \mathrm{~d}$ ), not because of impaired visual acuity and olfaction (Supplementary Figures S1F and G). These synaptic transmission deficits are similar to those observed in the neuromuscular junction of $d r p 1$-mutant Drosophila, ${ }^{34}$ which were attributed to a lack of synaptic ATP. We therefore measured oxygen consumption and ATP levels of hippocampal mitochondria and found oxygen consumption (Figure $5 \mathrm{e}$ ) as well as ATP content (Figure 5f) to be reduced in Drp1-deficient samples. In addition, ultrastructural analysis by transmission electron microscopy (TEM) revealed a significant reduction in presynaptic mitochondria of hippocampal $\operatorname{Drp} 1^{i \Delta b / i \Delta b}$ neurons (Figure $5 \mathrm{~g}$ ). The combination of reduced mitochondrial content in presynaptic terminals and defective ATP production can explain the impaired synaptic function observed in Drp $1^{i \Delta b / i \Delta b}$ mice.

Forebrain-specific neuronal Drp1 ablation leads to hippocampal atrophy. Several previous studies indicate that Drp1 ablation in vitro causes a reduction in the number of dendrites, spines, and synapses (reviewed by Oettinghaus et $a .^{28}$ ). However, as no substantial changes in dendritic morphology were observed early after Drp1 ablation (4 weeks p.t.i.; Figure 4a), we performed Golgi silver impregnations on $D r p 1^{i \Delta b / i \Delta b}$ brain sections 10 weeks p.t.i. Sholl analysis revealed that, although overall dendritic tree morphometry was unchanged, dendrites were shorter in $\operatorname{Drp} 1^{i \Delta b / \Delta b}$ mice (Figure 6a), which was associated with decreased 

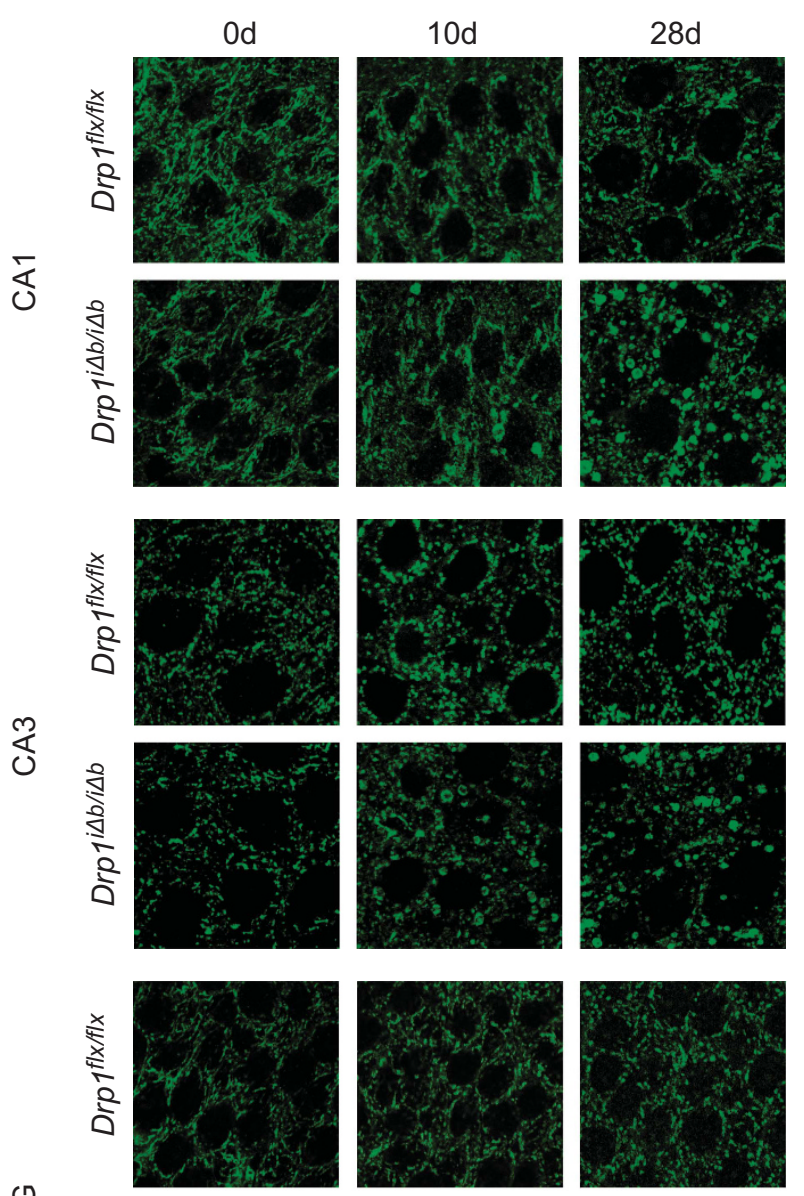

ֻั
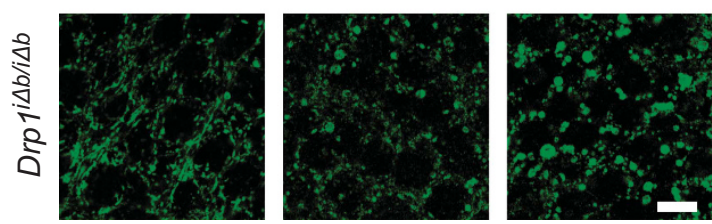

Figure 3 Mitochondrial morphology in the hippocampus after Drp1 ablation. Mice of the indicated genotype were killed at the indicated times p.ti. Coronal sections of formalin-fixed, paraffin-embedded brains of $\operatorname{Drp}^{i \Delta b / \Delta b}$ and control mice were immunostained for cytochrome $c$ oxidase subunit 1. Images show CA1 and CA3 pyramidal neurons and DG granule cells. Scale bar, $10 \mu \mathrm{m}$

hippocampal volume (Figure 6b). In contrast, cortical or midbrain volumes did not change significantly (Supplementary Figure $\mathrm{S} 1 \mathrm{H}$ ). To test whether this hippocampal atrophy was associated with loss of spines, we quantified spine numbers on apical dendritic trees of Golgi-impregnated pyramidal CA1 neurons but did not find any difference (Figure 6c). Likewise, the number of synapses, as assessed by co-localization of fluorescent stains for presynaptic (VGLUT2) and postsynaptic (PSD95) markers in confocal Z-stacks, was normal (Figure 6d). Thus, the depletion of mitochondria from presynaptic hippocampal neuron terminals can account for the observed synaptic transmission impairments and the specific deficits in spatial working memory upon Drp1 ablation. Nevertheless, the maintenance of normal synapse and spine numbers 10 weeks following Drp1 ablation indicates that mature hippocampal neurons are able to cope with blocked mitochondrial fission with only mild functional alterations.

Adult forebrain neurons do not degenerate within 10 weeks of Drp1 ablation. We next verified whether the above-reported changes caused by Drp1 ablation in the hippocampus led to neurodegeneration. Hippocampal neurons were qualitatively and quantitatively normal in hematoxylin-eosin (H\&E)-stained hippocampal tissue sections (Figures $7 \mathrm{a}$ and $\mathrm{b}$ ), with no signs of condensed eosinophilic neurons, chromatinolysis or apoptotic bodies. In addition, in situ TUNEL (TdT-mediated dUTP-biotin nick end labeling) did not detect any evidence of increased cell death in the hippocampus 10 weeks p.t.i. (Figures 7c and d). As neurons are believed to be especially dependent on ATP produced by oxidative phosphorylation, we wondered how they could compensate the reduced ATP production (Figure 5f) to avoid neurodegeneration. Comparative mass spectrometry analyses revealed that levels of glycolysis-related proteins were significantly changed, the rate-limiting enzyme Hexokinase 1 being markedly upregulated (4.37-fold; see Supplementary Table S1). These results indicate that neurons attempt to cope with the metabolic consequences of Drp1 ablation by upregulating glycolysis.

Although Drp1 ablation in Purkinje cells ${ }^{29}$ is accompanied by macroautophagy inhibition and reactive oxygen species (ROS) accumulation, extensive TEM analyses did not reveal significant autophagosome accumulation in the brain sections from $D r p 1^{i \Delta b / \Delta b}$ mice; of note, these consisted mostly of doublemembrane structures measuring $500 \mathrm{~nm}$ (i.e., too small to accommodate mitochondria; Supplementary Figure S2). Accordingly, comparative mass spectrometry in hippocampal neurons did not reveal significant changes in autophagy-related proteins, including p62 and LC3 (Supplementary Table S2). Moreover, levels of ROS (measured by dihydrorhodamine and MitoSox fluorescence on isolated mitochondria), of thiobarbituric acid-reactive substances (TBARS), indicators of lipid peroxidation, as well as of oxidized (versus total) glutathione, were unchanged in mouse brain homogenates 4 weeks p.t.i. (Figures 8a-d). Indeed, our comparative mass spectrometry analyses revealed that 11 out of the 48 detected oxidative stress-associated proteins were upregulated in $\operatorname{Drp} 1^{i \Delta b / i \Delta b}$ brains 10 weeks p.t.i. (Supplementary Table S3). Ten of these proteins are regulated by the transcription factor nuclear factor erythroid 2 related factor (NRF2), whose inhibitor KEAP1 (Kelch-like ECH-associated protein 1) was significantly downregulated (fold change: 0.38; $Q$ : 0.002; Supplementary Table S3). Among the upregulated proteins, we identified several glutathione $S$-transferases, participating in solubilizing peroxidized lipids and xenobiotics, and the multidrug resistance protein $1 \mathrm{~A}$ capable of removing toxic components from the cytosol. In contrast, cytosolic and mitochondrial thioredoxins, NRF2 target genes that help reducing oxidized protein, were downregulated. These results indicate that Drp1 ablation in postmitotic neurons leads to a moderate activation of cellular antioxidant systems, possibly explaining why ROS levels were not increased but testifying that indeed also in forebrain neurons Drp1 ablation leads to ROS production. As in Purkinje cells ROS are involved in the formation of enlarged spherical mitochondrial bodies (mitobulbs), ${ }^{29}$ we decided to analyze 
a
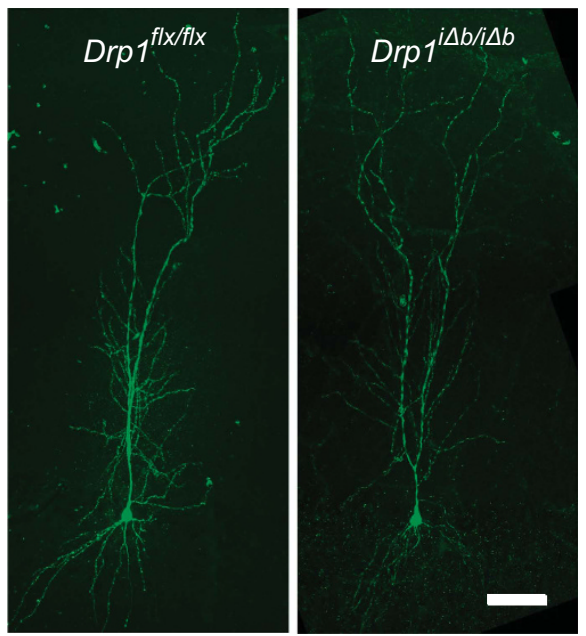

b
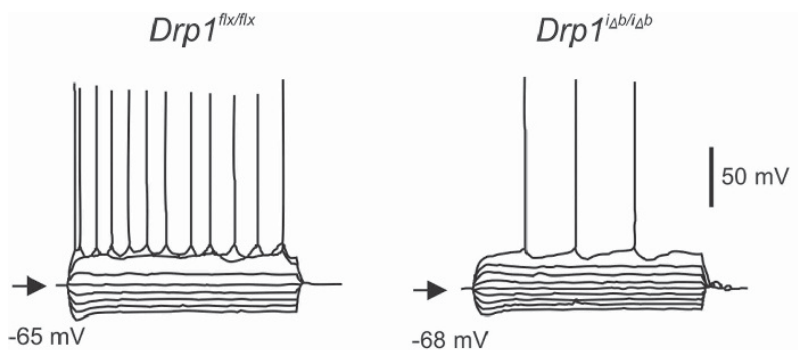

C

d
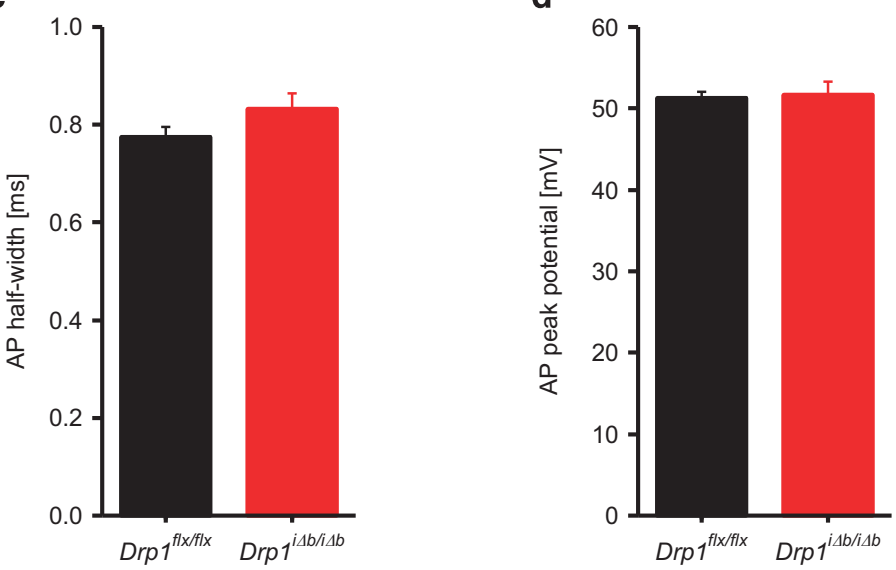

e

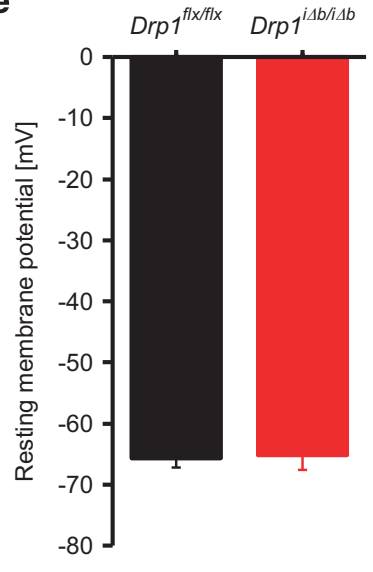

Figure 4 Electrophysiological properties are not affected in Drp1-ablated neurons. (a) Acute hippocampal slices of Drp1 $1^{i \Delta / \Delta b} 4$ weeks p.t.i. and control animals were prepared. CA1 pyramidal neurons were patch-clamped and infused with biocytin, which was revealed by immunofluorescence. Scale bar, $40 \mu \mathrm{m}$. Note the regular dendritic morphology. (b-d) One-second-long current steps of increasing amplitude were injected to induce action potentials in Drp $1^{i \Delta b / \Delta b} 4$ weeks p.t.i. and control CA1 pyramidal neurons; maximal amplitude and half width were plotted. Data represent average \pm S.E.M. of at least seven neurons. (e) Resting membrane potential of $D r p 1^{i \Delta b / \Delta b} 4$ weeks p.t.i. and control CA1 pyramidal neurons. Data represent average \pm S.E.M. of at least seven neurons

whether treatment of mice with the mitochondrially targeted antioxidant mito $\mathrm{Q}^{35}$ for 10 days p.t.i. could counteract the mitochondrial morphology phenotype. MitoQ significantly decreased mitobulbs in hippocampal CA1 and granule neurons of the DG (Figure 8e). However, in CA3 neurons transformation of mitochondria into mitobulbs is already complete at day 10 after Drp1 ablation (see Figure 3), and no mitoQ effect was observed, indicating that antioxidant treatment can merely delay the formation of mitobulbs but is not sufficient to suppress it.

In conclusion, unlike Purkinje cells, adult forebrain neurons respond to the increased ROS formation caused by Drp1 ablation by upregulating the cellular antioxidant defenses that partly neutralize ROS accumulation.

\section{Discussion}

Mitochondrial fragmentation is a hallmark of apoptosis and accordingly of several neurodegenerative disorders. Conversely, the dynamin-related GTPase Drp1 is indispensable for neuronal maturation and brain development ${ }^{15,16}$ and for cerebellar Purkinje cell survival. ${ }^{29}$ Our data conversely demonstrate that adult forebrain and hippocampal neurons display an increased functional reserve that allows them to survive Drp1 ablation.

In order to bypass the requirement for balanced mitochondrial dynamics during neuronal differentiation and brain development, ${ }^{15,16}$ we devised a model of acute, inducible Drp1 ablation in adult forebrain, including hippocampal neurons. Upon tamoxifen-induced Drp1 ablation, mitochondria appeared enlarged and perinuclearly confined. These neurons were unexpectedly able to cope well with Drp1 ablation: changes in neuronal morphology, oxidative stress and cell death were negligible, whereas the reduction in localized ATP supply to presynaptic terminals impaired synaptic transmission, resulting in early memory defects.

The first visible phenotype of $D r p 1^{i \Delta b / i \Delta b}$ mice, presenting as early as 4 weeks after tamoxifen administration, was a defect in spatial working memory, which could be traced back to the 


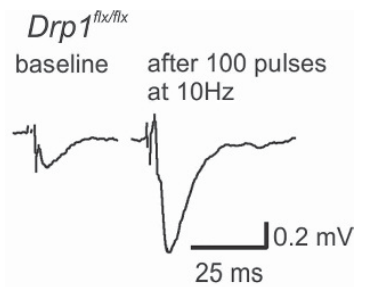

$\operatorname{Drp} 1^{i \Delta / / \Delta b}$

baseline after 100 pulses

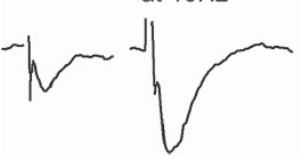

d
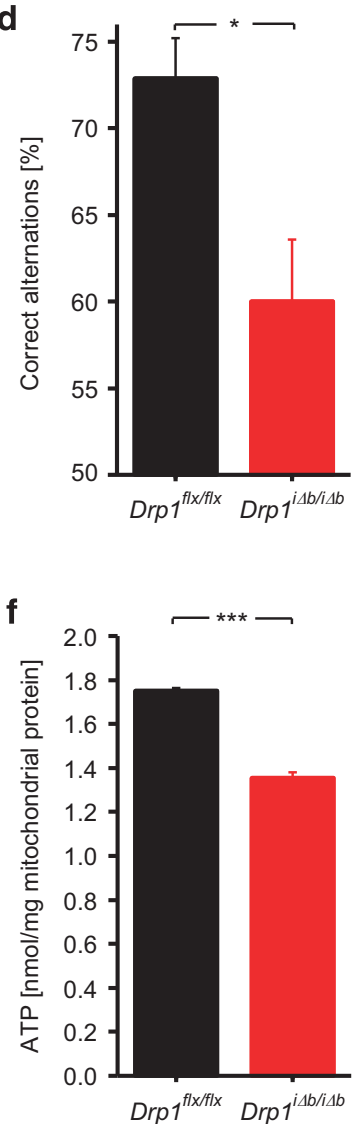

b

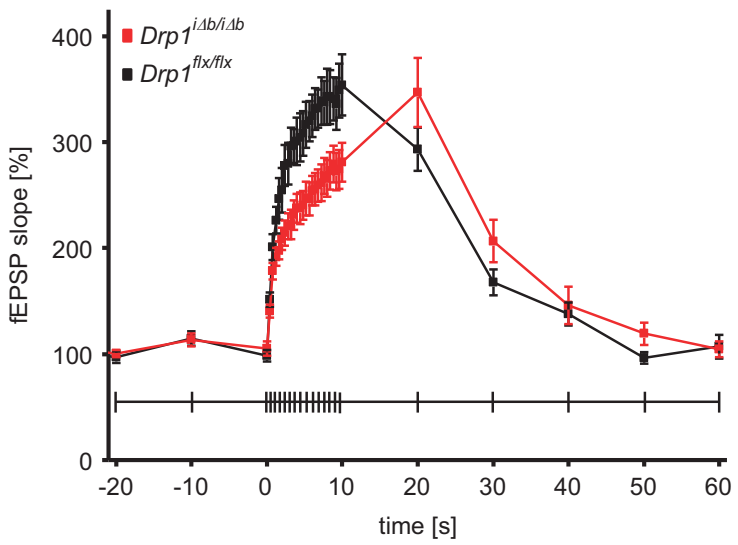

C

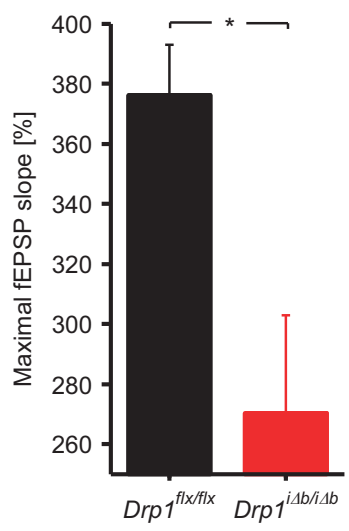

e

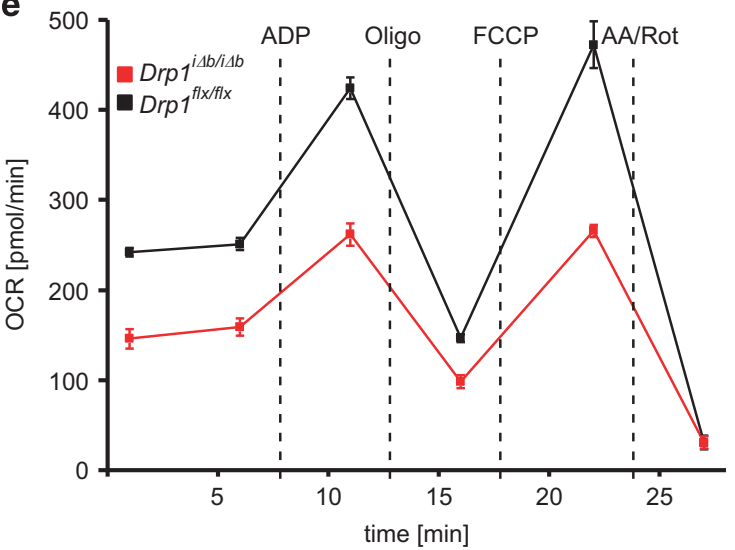

g

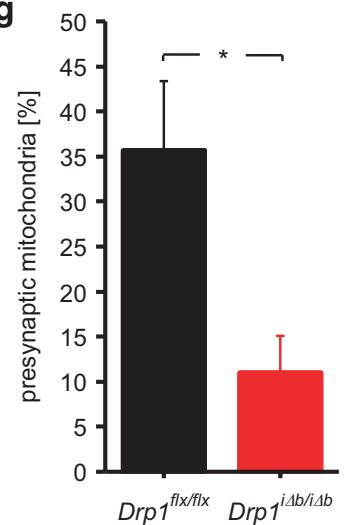

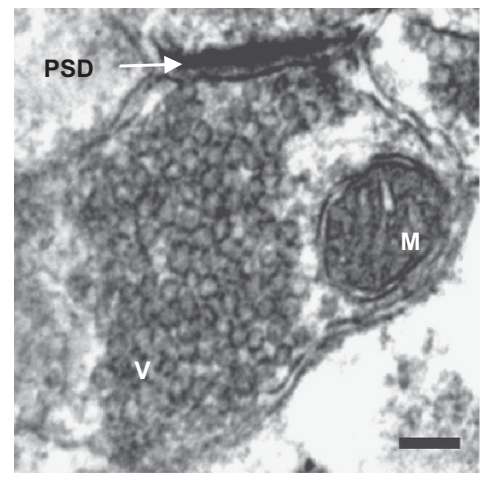

Figure 5 Impaired synaptic transmission in Drp1-ablated neurons. (a) Representative fEPSPs before and after $10 \mathrm{~s}$ of $10 \mathrm{~Hz}$ stimulation. fEPSPs were recorded in the CA1 stratum radiatum in Drp $1^{i \Delta / \Delta b}$ and control hippocampal slice cultures upon Schaffer collateral stimulation. (b) Mean slope of fEPSPs during the time course of a 10-s 10-Hz stimulation was plotted. Data represent average \pm S.E.M. of at least six neurons. (c) Maximal mean fEPSP slope after 10-s 10-Hz stimulation. Data represent average \pm S.E.M. of at least six neurons. (d) $D r p 1^{i \Delta b / \Delta b} 4$ weeks p.t.i. and control animals were placed in an eight-arm radial maze, which they were left to systematically explore. Correct alternation of arm visits was scored. Data represent average \pm S.E.M. of at least six animals. (e) Oxygen consumption rate of isolated hippocampal mitochondria of $D r p 1^{i \Delta b / \Delta b}$ and control mice was measured with a Seahorse Bioscience XF24 Analyzer. Substances were injected at the indicated time points. Data represent average \pm S.E.M. of at least four animals whose hippocampi were pooled; measurements performed with at least six replicates. (f) ATP content of isolated hippocampal mitochondria of $D r p 1^{i \Delta b / \Delta b}$ and control mice as measured with a bioluminescence assay. Data represent average \pm S.E.M. of at least four animals whose hippocampi were pooled; measurements were performed with at least eight replicates. (g) Synapses in TEM images of $\operatorname{Drp} 1^{i \Delta / \Delta b} 4$ weeks p.ti. and control hippocampi were screened for presynaptic mitochondria. Data represent average \pm S.E.M. of at least 4 animals of which at least 100 synapses each were screened. TEM image shows a representative synaptic structure with a presynaptic mitochondrion (M), presynaptic vesicles (V) and a postsynaptic dense area (PSD). Scale bar, $150 \mathrm{~nm}$. Asterisks denote $P$-values of an unpaired, two-tailed Student's $t$-test: ${ }^{*} P<0.05$ 
a

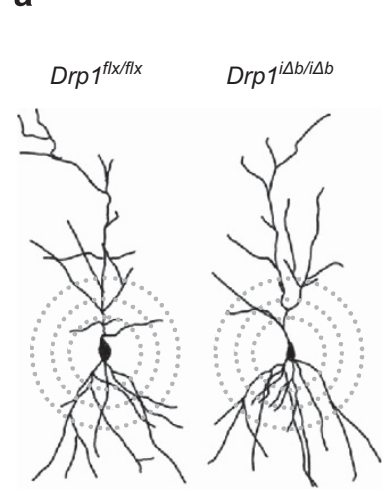

C

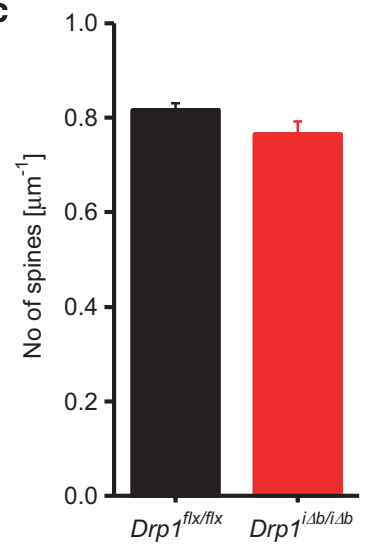

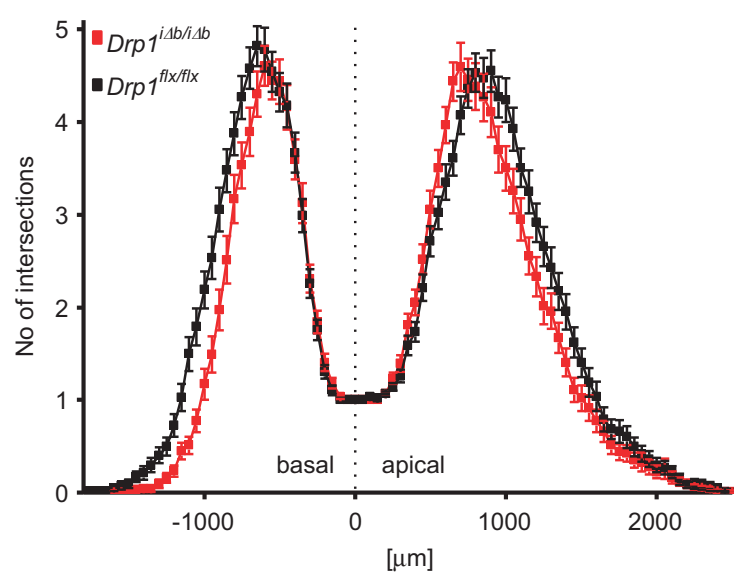

b

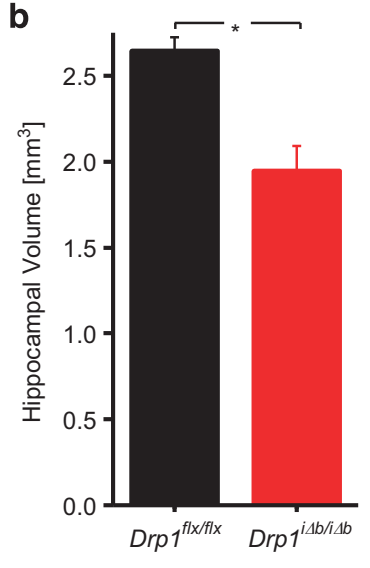

d
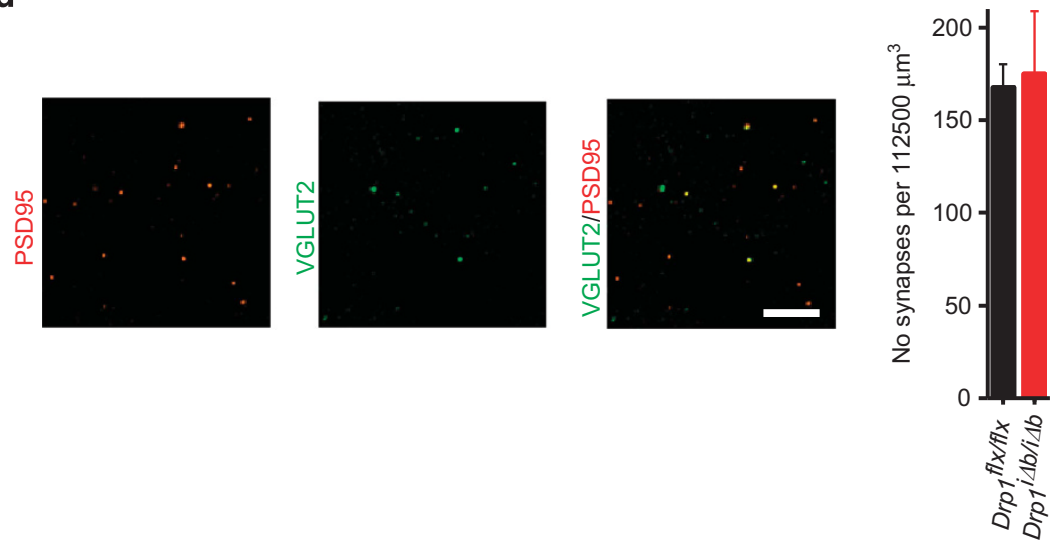

Figure 6 Long-term Drp1 ablation causes hippocampal atrophy. (a) Representative camera lucida drawings and Sholl analysis of Golgi-stained Drp $1^{i \Delta b / \Delta b} 10$ weeks p.t.i. and control CA1 pyramidal neurons. Each data point represents the number of intersections of the dendritic tree with concentric perisomatic rings of increasing diameter (exemplified by dashed circles). Data represent average \pm S.E.M. of at least 100 neurons. (b) Hippocampal volume was recorded on H\&E-stained, serial coronal cross-sections of $D r p 1^{i \Delta b / \Delta b}$ animals 8-weeks p.t.i. applying the Cavalieri principle. For cortical and midbrain volumes, only sections representing the coordinates bregma from 1.1 to $-1.2 \mathrm{~mm}$ were considered. Data represent average \pm S.E.M. of at least five animals. (c) Number of spines visualized by Golgi staining per $\mu \mathrm{m}$ dendrite length (apical dendritic tree of CA1 pyramidal neurons). Data represent average \pm S.E.M. of at least 3 animals of which 1000 spines were counted. (d) Coronal cross-sections of $D r p 1^{i \Delta b / \Delta b} 10$ weeks p.t.i. and control brains co-stained with presynaptic VGLUT2 (green) and postsynaptic marker PSD95 (red). A $150 \times 150 \times 5 \mu \mathrm{m}^{3}$ confocal image stack in the CA1 stratum radiatum was recorded, and the number of overlapping puncta was determined. Data represent average \pm S.E.M. of at least five animals of which four stacks each were recorded. Scale bar, $5 \mu \mathrm{m}$. Asterisks denote $P$-values of an unpaired, two-tailed Student's $t$-test: ${ }^{*} P<0.05$

impairment in synaptic transmission upon sustained stimulation. Accordingly, and in line with the fact that defects in mitochondrial fission impair mitochondrial transport in neurites, presynaptic mitochondria were decreased. A similar electrophysiological phenotype associated with abnormal mitochondrial morphology was observed at neuromuscular junctions of $d r p 1$ mutant flies. ${ }^{34}$ Although that phenotype has primarily been attributed to ATP-dependent effects on reserve pool vesicle recycling owing to the lack of mitochondria at synaptic boutons and the overall reduction in oxidative phosphorylation, DRP1 may also be directly involved in synaptic vesicle formation, as shown more recently in mouse hippocampal neurons. ${ }^{36}$ Resting electrophysiological properties as well as paired-pulse facilitation, which were all unaffected, argue against a contribution of calcium-buffering defects to the overall phenotype (recently reviewed in Williams et al. ${ }^{37}$ ).

A predictable consequence of inhibiting mitochondrial fission was the change in mitochondrial network morphology, with predominantly enlarged spherical mitochondria that clustered around the nucleus. Of note, while previous reports in Purkinje cells ${ }^{29}$ attributed these 'mitobulbs' to oxidative stress ensuing from defects in autophagy, we did not detect evidence suggesting a significant blockage of the latter in vivo, nor the presence of oxidative markers. Therefore, our data indicate that neurons in vivo are supported by the brain milieu to keep autophagy at a minimum level, even under stress conditions. This is consistent with reports that autophagy is an extremely rare event in neurons in vivo, even after $48 \mathrm{~h}$ of starvation. ${ }^{38}$ Even so, buffering of oxygen radicals by mitoQ administration slowed down the formation of 'mitobulbs' in our mice, suggesting that an oxidative stress component is present in Drp1-ablated neurons, but efficiently managed by cellular defenses. A possible explanation for this discrepancy is that Purkinje cells represent an exceptionally large and extensively connected neuronal subtype, which might entail very high metabolic activity and associated ROS production (reviewed in Kern and Jones ${ }^{39}$ ), all of which could contribute to 

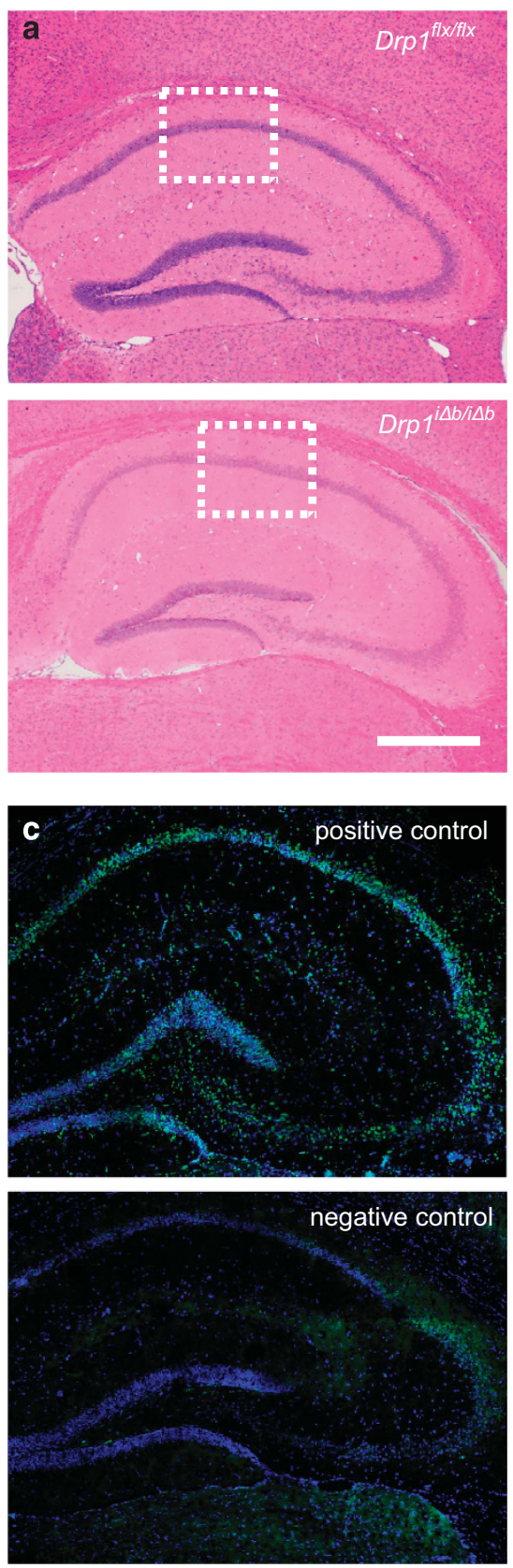
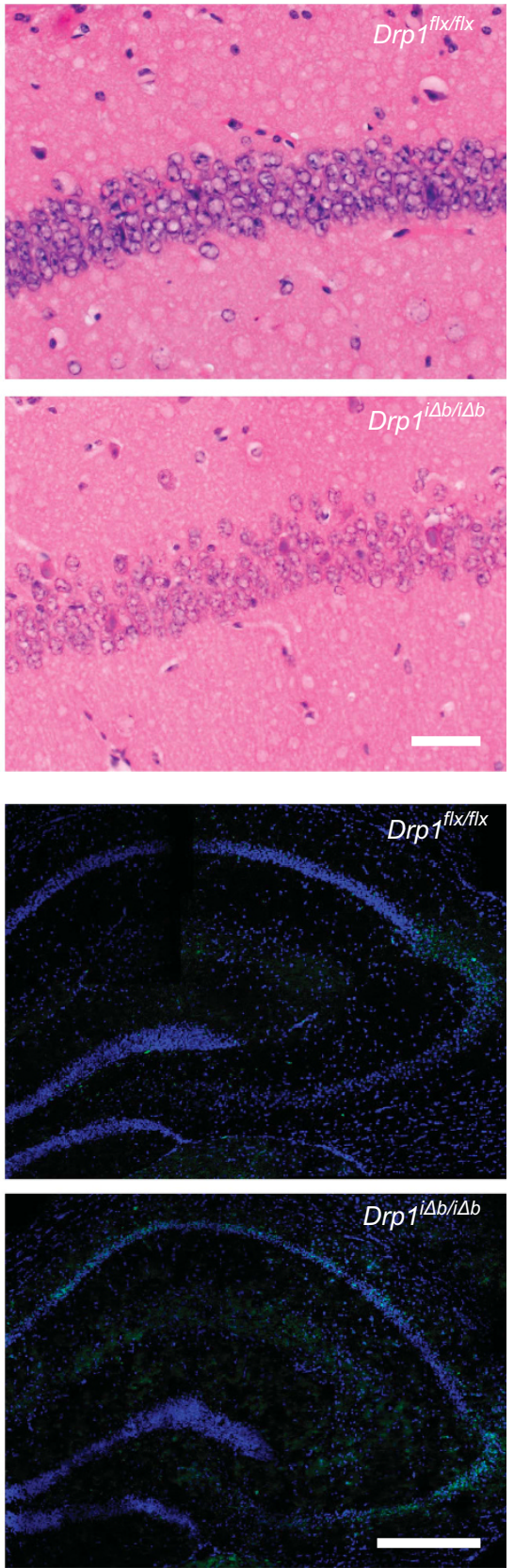
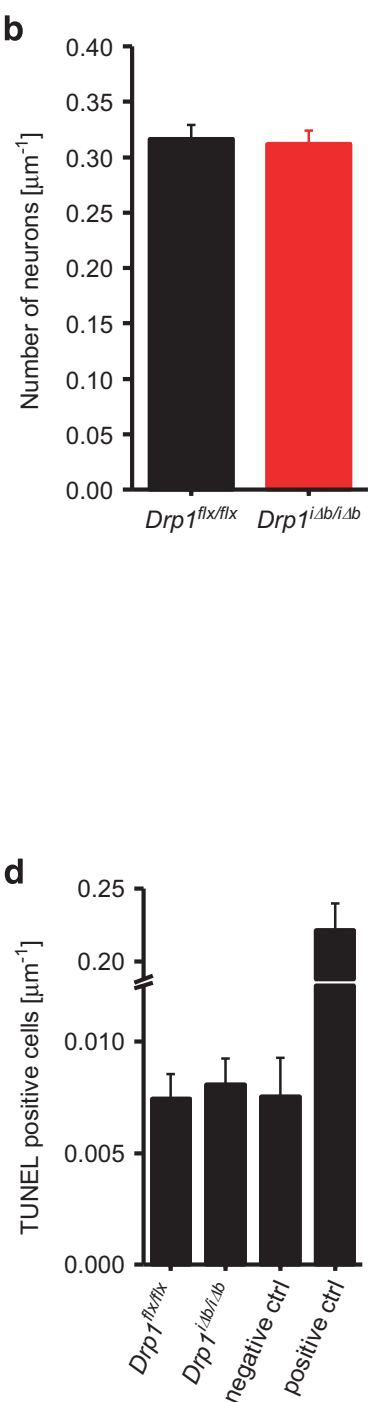

Figure 7 Drp1 ablation does not lead to hippocampal neurodegeneration. (a) H\&E staining of coronal cross-sections of formalin-fixed, paraffin-embedded Drp $1^{i \Delta b / \Delta b}$ 10 weeks p.t.i. and control brains showing the hippocampus and a magnification of the CA1 region. Scale bars, $1 \mathrm{~mm}$ (left) and $50 \mu \mathrm{m}$ (right). (b) Neuronal nuclei in the CA1 region were quantified manually. Data represent average \pm S.E.M. of at least 5 animals of which at least 200 nuclei each were counted. (c) In situ TUNEL of coronal cross-sections of frozen Drp $1^{i \Delta b / \Delta b} 10$ weeks p.ti. and control brains showing hippocampus. Positive control was preincubated with DNAse I; negative control incubated without terminal deoxynucleotidyl transferase. Scale bar, $1 \mathrm{~mm}$. (d) Neuronal nuclei in the CA1 region were quantified over a 250- $\mu \mathrm{m}$ length. Data represent average \pm S.E.M. of at least five animals

selective vulnerability of this neuronal subtype under certain stress conditions. It is important to note that Purkinje cell death in vivo started to occur already at around week 9 after Drp1 ablation. ${ }^{29}$ On the other hand, in line with the absence of oxidative stress, no neurodegeneration was observed upon 10-week-long Drp1 ablation in the hippocampus, the time frame when $D r p 1^{i \Delta b / i \Delta b}$ animals had to be killed due to profound systemic-level metabolic changes (manuscript in preparation). Therefore, it cannot be excluded that the impaired neuronal mitochondrial functions might culminate in hippocampal neuronal death occurring 3 months beyond Drp1 ablation. Additionally, it is worth noting that murine Purkinje neuron differentiation continues throughout the first 3 weeks of life, ${ }^{40}$ the time when the $L 7 / p c p 2$ promoter used by Kageyama et $a l^{29}$ starts to be active. It may therefore be speculated that Drp1 ablation in these neurons may still overlap with the final stages of murine cerebellar development and thus also contribute to the observed Purkinje cell degeneration. 
a

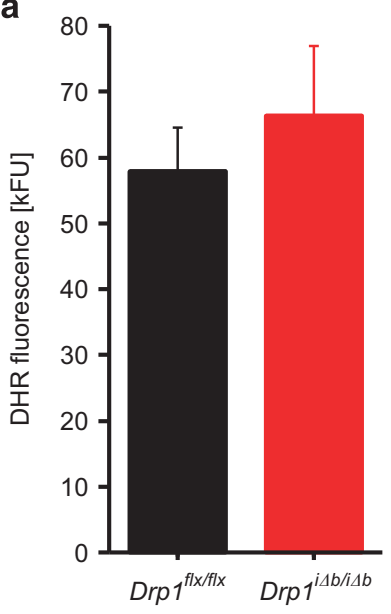

b

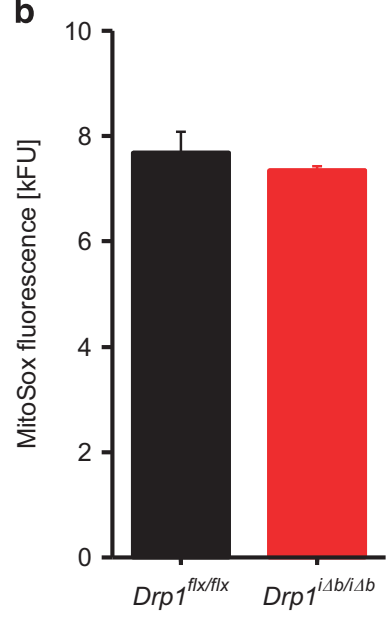

c

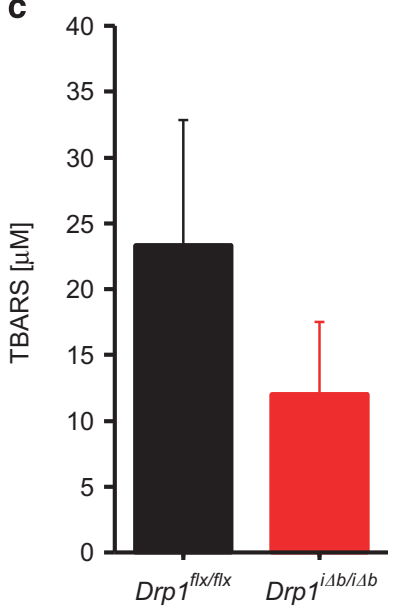

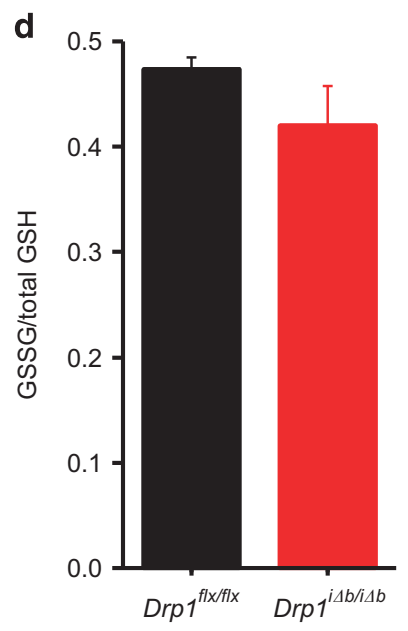
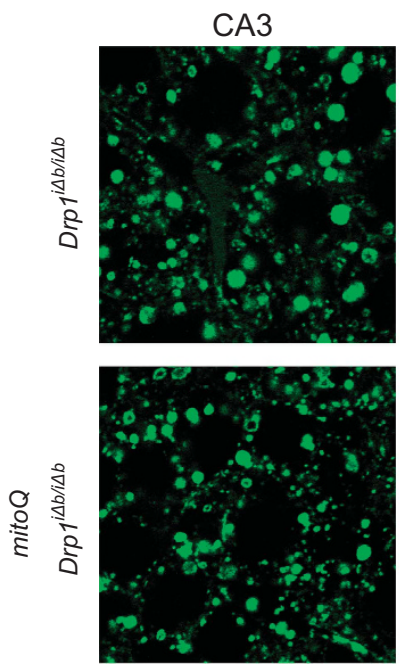
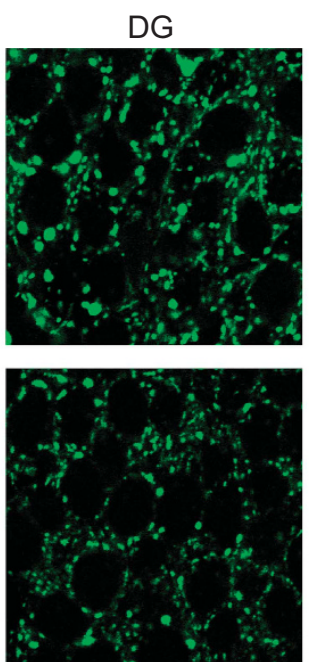
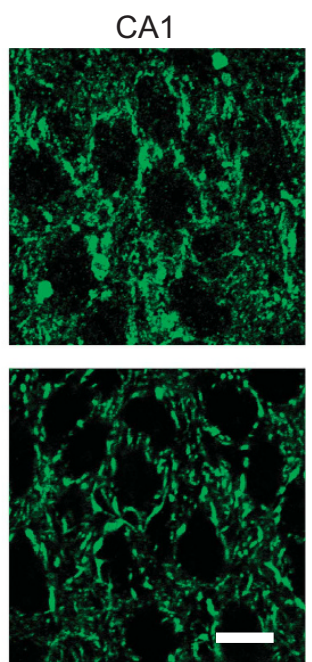

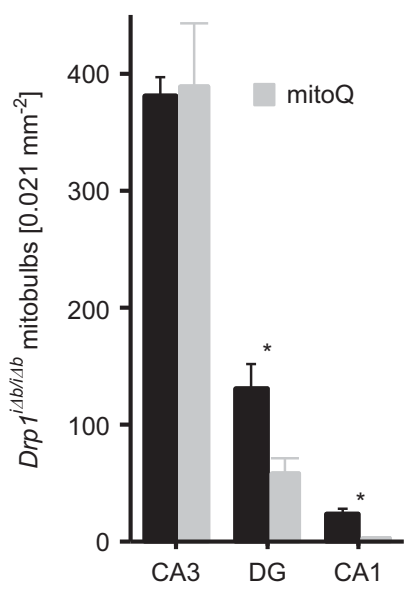

Figure 8 The antioxidant mitoQ ameliorates mitochondrial morphology in Drp1-deficient CA1 and DG hippocampal neurons. (a and $\mathbf{b}$ ) Oxidative stress levels represented by dihydrorhodamine (DHR) and MitoSox fluorescence in cortical and hippocampal tissue homogenates of $D r p 1^{\Delta b / \Delta b} 4$ weeks p.ti. and control mice. Data represent average \pm S.E.M. of at least four animals (tissue pooled), and measurements were performed with a minimum of four replicates. (c) Thiobarbituric acid-reactive substance (TBARS) levels, reflecting lipid peroxidation, in $\operatorname{Drp} 1^{\Delta b / \Delta b} 4$ weeks p.ti. and control cortical and hippocampal lysates measured by colorimetric assay. Data represent average \pm S.E.M. of at least six animals. (d) Ratio of oxidized to total glutathione in hippocampal lysates of $D r p 1^{i \Delta b / \Delta b} 4$ weeks p.t.i. and control mice measured by colorimetric assay. Data represent average \pm S.E.M. of at least six animals. (e) $D r p 1^{i b / \Delta b}$ animals treated or not with daily mitoQ injections of $20 \mathrm{mg} / \mathrm{kg}$ i.p. were killed 10 days p.ti... and coronal cross-sections of formalin-fixed, paraffin-embedded brains immunostained for cytochrome coxidase subunit 1. Mitobulbs of randomly selected areas within the indicated hippocampal subregions were quantified. Scale bar, $10 \mu \mathrm{m}$. Data represent average \pm S.E.M. of at least four animals. Asterisks denote $P$-values of an unpaired, two-tailed Student's t-test. ${ }^{*} P<0.05$

We can conversely exclude neurodevelopmental defects caused by Drp1 ablation in our model, as recombination was induced at the age of 8 weeks and dendritic tree morphology as well as synapse and spine numbers were unaffected. Of note, a recent study reporting neurodegeneration upon Drp1 ablation in postmitotic dopaminergic neurons supports the notion that sensitivity to Drp1 ablation is neuronal subtypedependent. $^{41}$

Collectively, our results indicate that adult hippocampal neurons cope with profound impairment of mitochondrial dynamics and function by activating antioxidant and metabolic compensatory mechanisms. Predictably, certain neuronal functions, such as synaptic transmission, can be affected due to depletion of the organelles from presynaptic terminals, culminating in memory deficits. Conversely, these compensatory circuits are sufficient to maintain overall neuronal morphology, synapse and spine numbers and ultimately viability for as long as 3 months following Drp1 ablation. Our data therefore indicate that forebrain neurons can resist to mitochondrial dysmorphology and dysfunction, a critical feature to protect them and therefore the cognitive functions of higher mammals from mitochondriotoxic stimuli.

\section{Materials and Methods}

Mice. Drpt ${ }^{f l x f l x}$ mice ${ }^{15}$ were crossed with CaMKIlla CreERT2 $\left(\mathrm{Cre}^{+}\right)$animals, obtained from the European Mouse Mutant Archive (EMMA strain 02125). ${ }^{33}$ At 8 weeks of age, the resulting $\operatorname{Drpt}^{f^{f f} f f l x} \mathrm{Cre}^{+}$mice were injected i.p. with $1 \mathrm{mg}$ tamoxifen (Sigma (Buchs, Switzerland); $10 \mathrm{mg} / \mathrm{ml}$ tamoxifen dissolved in a 9:1 ratio of sunflower seed oil to ethanol) twice daily on 5 consecutive days to induce recombination of the Drp1 locus. To check for recombination, Drp $1^{\text {fliffx }} \mathrm{Cre}{ }^{+}$mice were crossed with the RCE:loxP reporter mouse strain (Jackson Laboratories, Bar Harbor, ME, USA; strain 032037-JAX) harboring the R26R CAG-boosted Egfp (RCE) allele with a floxed STOP cassette upstream of the Egfp gene. 
Histology. Serial coronal cross-sections of formalin-fixed, paraffin-embedded mouse brains were prepared, and sections representing the coordinates bregma $(-1.34 \mathrm{~mm})-(-2.46 \mathrm{~mm})$ were selected and stained for COX subunit 1a (Abcam, Cambridge, UK, ab14705). Z-stacks were recorded with an inverted Zeiss (Feldbach, Switzerland) Axiovert 200M LSM 510 Meta confocal microscope with a $\times 100 / 1.4$ Oil DIC objective using Enterprise (Zuerich, Switzerland) $405 \mathrm{~nm}$ and Argon $488 \mathrm{~nm}$ lasers. Z-stacks were projected onto a single plane using ImageJ $(\mathrm{NIH}$, Bethesda, MD, USA). Synapse numbers were quantified as described previously. ${ }^{42}$ Golgi staining was performed on PBS-perfused, unfixed brains using a commercial kit (FD Neurotechnologies, Columbia, MD, USA).

Hippocampal volume was calculated from H\&E-stained $100-\mu \mathrm{m}$-spaced, serial coronal cross-sections applying the Cavalieri principle. For cortical and midbrain volume, only sections representing coordinates bregma from 1.1 to $-1.2 \mathrm{~mm}$ were considered, using corpus callosum as reference. TUNEL staining was performed on frozen, PBS-perfused, fixed brains using a commercial kit (Roche, Basel, Switzerland). Confocal images were stitched using a Fiji Plugin. ${ }^{43}$

Transmission electron microscopy. Semithin sections of osmiumstained hippocampi were prepared in order to identify hippocampal neurons, of which ultrathin sections were prepared. Imaging was done on a Phillips (Zürich, Switzerland) CM100 transmission electron microscope. Randomly selected TEM images were used to quantify presynaptic mitochondria of hippocampal synapses.

Electrophysiology. Transverse $350-400 \mu$ m-thick hippocampal brain slices were cut in a sucrose-based solution. During electrophysiological recordings, slices were continuously superfused with artificial cerebrospinal fluid maintained at $32-33^{\circ} \mathrm{C}$. During whole-cell patch-clamp recordings, hippocampal CA1 pyramidal neurons were filled with biocytin for subsequent morphological evaluation. fEPSPs were recorded with glass pipettes filled with $1 \mathrm{M} \mathrm{NaCl}$ placed in the stratum radiatum of the CA1 region. The stimulating electrode was placed $\sim 500 \mu \mathrm{m}$ away to stimulate Schaffer collaterals. Data analysis was performed offline using customized scripts written in python and Stimfit.

Behavioral analyses. Visual performance was tested in a water tank with a visible platform. Olfaction was checked using the cookie finding test. To score hippocampus-dependent working memory, the spontaneous alternation task was employed in an eight-arm radial maze, based on the spontaneous alternation paradigm. $^{44}$

Western blotting. Proteins of brain lysates were separated on 4-12\% BisTris SDS-PAGE gels, blotted onto PVDF membranes using the iBlot Dry Blotting System (Life Technologies, Carlsbad, CA, USA) and probed with the indicated primary antibodies and isotype-matched secondary antibodies conjugated to horseradish peroxidase. Signals were detected using ECL (GE Healthcare, Chalfont St Giles, UK).

Tissue culture. Cortical neurons from Drp1 ${ }^{f f x f f x}$ mice were prepared from E16.5 embryos by modifying a previously described method. ${ }^{45}$ Prior to plating, cells were electroporated using the Neon Electroporation System (Life Technologies). For complete protocol, please refer to the Supplementary Information.

Quantitative proteomics. Brain tissue samples were lysed in $2 \%$ sodium deoxycholate, $100 \mathrm{mM}$ ammonium bicarbonate buffer, reduced with $5 \mathrm{mM}$ tris(2-chlorethyl)phosphate and alkylated with $10 \mathrm{mM}$ iodoacetamide. Proteins were then digested by incubation with sequencing-grade modified trypsin $(1 / 50, \mathrm{w} / \mathrm{w}$; Promega, Madison, WI, USA) overnight at $37^{\circ} \mathrm{C}$. The digested samples were subsequently labeled with isobaric tag (TMT 6-plex, Thermo Fisher Scientific, Waltham, MA, USA) according to the manufacturer's instructions. The TMT-labeled samples were re-solubilized to a final concentration of $1 \mathrm{mg} / \mathrm{ml}$ and separated on a 12-cm pH 3-10 IPG strip (GE Healthcare) with a 3100 OFFGEL fractionator (Agilent, Basel, Switzerland) as previously described. ${ }^{46}$

The setup of the $\mu$ RPLC-MS system was as described previously. ${ }^{47}$ Chromatographic separation of peptides was carried out using an EASY nano-LC 1000 system (Thermo Fisher Scientific), equipped with a heated RP-HPLC column $(75 \mu \mathrm{m} \times 50 \mathrm{~cm})$ packed in-house with $1.9 \mu \mathrm{m} \mathrm{C18}$ resin (Reprosil-AQ Pur, Dr. Maisch). Mass spectrometry analysis was performed on a dual pressure LTQ-Elite Orbitrap mass spectrometer equipped with a nanoelectrospray ion source (both Thermo Fisher Scientific).

Using the MASCOT algorithm (Matrix Science, Version 2.4.0), the resulting mgf files were searched against a decoy database containing normal and reverse sequences of the predicted SwissProt entries of Mus musculus (www.ebi.ac.uk, release date 16/05/2012) and commonly observed contaminants (in total 33832 sequences for Mus musculus) generated using the SequenceReverser tool from the MaxQuant software (Version 1.0.13.13; www.maxquant.org). Next, database search results were imported to the Scaffold Q+ software (version 4.3.3, Proteome Software Inc., Portland, OR, USA), and the protein false identification rate was set to $1 \%$ based on the number of decoy hits. Protein probabilities were assigned by the Protein Prophet program. ${ }^{48}$ Acquired reporter ion intensities in the experiments were employed for automated quantification and statistically analyzed using a modified version of our in-house developed SafeQuant $R$ script. ${ }^{47}$ In brief, reporter ion intensities were corrected for isotopic impurities according to the manufacturer's instructions. Intensities for each peptide and protein ID were summed, globally normalized across all acquisition runs and employed for ratio calculation and statistical analysis. For complete mass spectrometry materials and methods, please refer to the Supplementary Information.

Oxygen consumption and ATP levels. Mitochondria were isolated from hippocampus as previously described. ${ }^{49}$ Oxygen consumption rate was measured in isolated mitochondria from cortex and hippocampus using a Seahorse Bioscience (North Billerica, MA, USA) XF24Analyzer. ATP content from isolated mitochondria was determined by a bioluminescence assay (ViaLighTM HT; Cambrex Bio Science, Lonza, Basel, Switzerland).

\section{Conflict of Interest}

The authors declare no conflict of interest.

Acknowledgements. We thank M Rüegg (Biozentrum, University of Basel) for helpful scientific discussions and advice; and C Lautenschlager, M Bänziger, S Ipsen (all from Institute of Pathology, Basel University Hospital) and M Dürrenberger (Microscopy Center Biozentrum, University of Basel) for their expert help with experimental procedures. Mito $Q$ was kindly provided by M Murphy (MRC, Cambridge UK); pPGK-Cre was a kind gift from T Langer (Cologne). This work was supported by Swiss National Science Foundation grant 31003A_127308, the Novartis Foundation for Medical-Biological Research, the Desirée and Nils Yde Foundation (420-14) and the Nora van Meeuwen-Haefliger Foundation (to SF), a grant from the Forschungsfonds of Basel University (to BO) and Telethon Italy GGP12162, GPP10005, AIRC Italy, ERC ERMITO, FP7 CIG CristOpa, MIUR FIRB Automed (to LS). Publication costs are supported by the Neurex network (TriNeuron - Program Interreg IV Upper Rhine - European Regional Development Fund) www.neurex.org.

\section{Author contributions}

BO, JMS, LMR, ML, CS, KS, AG and LM performed experiments. JB, AS, JH, AE, $P D ' A, P F$ and MT analyzed and interpreted experimental data. NI and KM provided reagents. LS and SF conceived the project, coordinated and supervised research. BO, LMR, LS and SF wrote the manuscript.

1. Nunnari J, Suomalainen A. Mitochondria: in sickness and in health. Cell 2012; 148 1145-1159.

2. Sheng Z-H. Mitochondrial trafficking and anchoring in neurons: New insight and implications. J Cell Biol 2014; 204: 1087-1098.

3. McLelland G-L, Soubannier V, Chen CX, McBride HM, Fon EA. Parkin and PINK1 function in a vesicular trafficking pathway regulating mitochondrial quality control. EMBO J 2014; 33: 282-295.

4. Twig G, Elorza A, Molina AJ, Mohamed H, Wikstrom JD, Walzer G et al. Fission and selective fusion govern mitochondrial segregation and elimination by autophagy. EMBO $J$ 2008; 27: 433-446.

5. Chen $\mathrm{H}$, Chomyn A, Chan DC. Disruption of fusion results in mitochondrial heterogeneity and dysfunction. J Biol Chem 2005; 280: 26185-26192.

6. Bliek AM, van der, Shen Q, Kawajiri S. Mechanisms of mitochondrial fission and fusion. Cold Spring Harb Perspect Biol 2013; 5: a011072.

7. Koshiba T, Detmer SA, Kaiser JT, Chen H, McCaffery JM, Chan DC. Structural basis of mitochondrial tethering by mitofusin complexes. Science 2004; 305: 858-862.

8. Cipolat S, de Brito OM, Dal Zilio B, Scorrano L. OPA1 requires mitofusin 1 to promote mitochondrial fusion. Proc Natl Acad Sci USA 2004; 101: 15927-15932.

9. De Brito OM, Scorrano L. Mitofusin 2 tethers endoplasmic reticulum to mitochondria. Nature 2008; 456: 605-610.

10. Cereghetti GM, Stangherlin A, Martins de Brito O, Chang CR, Blackstone C, Bernardi $P$ et al. Dephosphorylation by calcineurin regulates translocation of Drp1 to mitochondria. Proc Natl Acad Sci USA 2008; 105: 15803-15808. 
11. Otera H, Wang C, Cleland MM, Setoguchi K, Yokota S, Youle RJ et al. Mff is an essential factor for mitochondrial recruitment of Drp1 during mitochondrial fission in mammalian cells. J Cell Biol 2010; 191: 1141-1158.

12. Friedman JR, Lackner LL, West M, DiBenedetto JR, Nunnari J, Voeltz GK. ER tubules mark sites of mitochondrial division. Science 2011; 334: 358-362.

13. Bui HT, Shaw JM. Dynamin assembly strategies and adaptor proteins in mitochondrial fission. Curr Biol 2013; 23: R891-R899.

14. Waterham HR, Koster J, van Roermund CW, Mooyer PA, Wanders RJ, Leonard JV. A lethal defect of mitochondrial and peroxisomal fission. N Engl J Med 2007; 356: 1736-1741.

15. Ishihara N, Nomura M, Jofuku A, Kato H, Suzuki SO, Masuda K et al. Mitochondrial fission factor Drp1 is essential for embryonic development and synapse formation in mice. Nat Cell Biol 2009; 11: 958-966.

16. Wakabayashi J, Zhang Z, Wakabayashi N, Tamura Y, Fukaya M, Kensler TW et al. The dynamin-related GTPase Drp1 is required for embryonic and brain development in mice. J Cell Biol 2009; 186: 805-816.

17. Barsoum MJ, Yuan H, Gerencser AA, Liot G, Kushnareva Y, Graber S et al. Nitric oxideinduced mitochondrial fission is regulated by dynamin-related GTPases in neurons. EMBO J 2006; 25: 3900-3911.

18. Cheung EC, McBride HM, Slack RS. Mitochondrial dynamics in the regulation of neuronal cell death. Apoptosis 2007; 12: 979-992.

19. Costa V, Giacomello M, Hudec R, Lopreiato R, Ermak G, Lim D et al. Mitochondrial fission and cristae disruption increase the response of cell models of Huntington's disease to apoptotic stimuli. EMBO Mol Med 2010; 2: 490-503.

20. Dagda RK, Merrill RA, Cribbs JT, Chen Y, Hell JW, Usachev YM et al. The spinocerebellar ataxia 12 gene product and protein phosphatase $2 A$ regulatory subunit Bbeta2 antagonizes neuronal survival by promoting mitochondrial fission. J Biol Chem 2008; 283: 36241-36248.

21. Meuer K, Suppanz IE, Lingor P, Planchamp V, Goricke B, Fichtner L et al. Cyclin-dependent kinase 5 is an upstream regulator of mitochondrial fission during neuronal apoptosis. Cell Death Differ 2007; 14: 651-661.

22. Tian C, Murrin LC, Zheng JC. Mitochondrial fragmentation is involved in methamphetamineinduced cell death in rat hippocampal neural progenitor cells. PLoS One 2009; 4: e5546.

23. Young KW, Pinon LG, Bampton ET, Nicotera P. Different pathways lead to mitochondrial fragmentation during apoptotic and excitotoxic cell death in primary neurons. J Biochem $\mathrm{Mo}$ Toxicol 2010; 24: 335-341.

24. Yuan H, Gerencser AA, Liot G, Lipton SA, Ellisman M, Perkins GA et al. Mitochondrial fission is an upstream and required event for bax foci formation in response to nitric oxide in cortical neurons. Cell Death Differ 2007; 14: 462-471.

25. Lee YJ, Jeong SY, Karbowski M, Smith CL, Youle RJ. Roles of the mammalian mitochondrial fission and fusion mediators Fis1, Drp1, and Opa1 in apoptosis. Mol Biol Cell 2004; 15: 5001-5011.

26. Uo T, Dworzak J, Kinoshita C, Inman DM, Kinoshita Y, Horner PJ et al. Drp1 levels constitutively regulate mitochondrial dynamics and cell survival in cortical neurons. Exp Neurol 2009; 218: 274-285.

27. Wang X, Su B, Lee HG, Li X, Perry G, Smith MA et al. Impaired balance of mitochondrial fission and fusion in Alzheimer's disease. J Neurosci 2009; 29: 9090-9103.

28. Oettinghaus B, Licci M, Scorrano L, Frank S. Less than perfect divorces: dysregulated mitochondrial fission and neurodegeneration. Acta Neuropathol 2012; 123: 189-203.

29. Kageyama Y, Zhang Z, Roda R, Fukaya M, Wakabayashi J, Wakabayashi N et al. Mitochondrial division ensures the survival of postmitotic neurons by suppressing oxidative damage. J Cell Biol 2012; 197: 535-551.

30. Grohm J, Kim S-W, Mamrak U, Tobaben S, Cassidy-Stone A, Nunnari J et al. Inhibition of Drp1 provides neuroprotection in vitro and in vivo. Cell Death Differ 2012; 19: 1446-1458.

31. Park SW, Kim KY, Lindsey JD, Dai Y, Heo H, Nguyen DH et al. A selective inhibitor of drp1, mdivi-1, increases retinal ganglion cell survival in acute ischemic mouse retina. Invest Ophthalmol Vis Sci 2011; 52: 2837-2843.
32. Zhang N, Wang S, Li Y, Che L, Zhao Q. A selective inhibitor of Drp1, mdivi-1, acts against cerebral ischemia/reperfusion injury via an anti-apoptotic pathway in rats. Neurosci Lett 2013; 535: 104-109.

33. Erdmann G, Schutz G, Berger S. Inducible gene inactivation in neurons of the adult mouse forebrain. BMC Neurosci 2007; 8: 63.

34. Verstreken P, Ly CV, Venken KJ, Koh TW, Zhou Y, Bellen HJ. Synaptic mitochondria are critical for mobilization of reserve pool vesicles at Drosophila neuromuscular junctions. Neuron 2005; 47: 365-378.

35. Kelso GF, Porteous CM, Coulter CV, Hughes G, Porteous WK, Ledgerwood EC et al. Selective targeting of a redox-active ubiquinone to mitochondria within cells antioxidant and antiapoptotic properties. J Biol Chem 2001; 276: 4588-4596.

36. Li H, Alavian KN, Lazrove E, Mehta N, Jones A, Zhang P et al. A Bcl-xL-Drp1 complex regulates synaptic vesicle membrane dynamics during endocytosis. Nat Cell Biol 2013; 15 : 773-785.

37. Williams GSB, Boyman L, Chikando AC, Khairallah RJ, Lederer WJ. Mitochondrial calcium uptake. Proc Natl Acad Sci USA 2013; 110: 10479-10486.

38. Mizushima N, Yamamoto A, Matsui M, Yoshimori T, Ohsumi Y. In vivo analysis of autophagy in response to nutrient starvation using transgenic mice expressing a fluorescent autophagosome marker. Mol Biol Cell 2004; 15: 1101-1111.

39. Kern JK, Jones AM. Evidence of toxicity, oxidative stress, and neuronal insult in autism. $J$ Toxicol Environ Health B Crit Rev 2006; 9: 485-499.

40. Sudarov A, Joyner AL. Cerebellum morphogenesis: the foliation pattern is orchestrated by multi-cellular anchoring centers. Neural Dev 2007; 2: 26.

41. Berthet A, Margolis EB, Zhang J, Hsieh I, Zhang J, Hnasko T et al. Loss of mitochondrial fission depletes axonal mitochondria in midbrain dopamine neurons. J Neurosci 2014.

42. Ippolito DM, Eroglu C. Quantifying Synapses: an immunocytochemistry-based assay to quantify synapse number. J Vis Exp 2010: 2270.

43. Preibisch S, Saalfeld S, Tomancak P. Globally optimal stitching of tiled 3D microscopic image acquisitions. Bioinformatics 2009; 25: 1463-1465.

44. Ragozzino ME, Unick KE, Gold PE. Hippocampal acetylcholine release during memory testing in rats: augmentation by glucose. Proc Natl Acad Sci USA 1996; 93: 4693-4698.

45. Abramov AY, Scorziello A, Duchen MR. Three distinct mechanisms generate oxygen free radicals in neurons and contribute to cell death during anoxia and reoxygenation. $J$ Neurosci 2007; 27: 1129-1138.

46. Beck M, Schmidt A, Malmstroem J, Claassen M, Ori A, Szymborska A et al. The quantitative proteome of a human cell line. Mol Syst Biol 2011; 7: 549.

47. Glatter T, Ludwig C, Ahrné E, Aebersold R, Heck AJR, Schmidt A. Large-scale quantitative assessment of different in-solution protein digestion protocols reveals superior cleavage efficiency of tandem Lys-C/trypsin proteolysis over trypsin digestion. J Proteome Res 2012; 11: $5145-5156$.

48. Nesvizhskii Al, Keller A, Kolker E, Aebersold R. A statistical model for identifying proteins by tandem mass spectrometry. Anal Chem 2003; 75: 4646-4658.

49. Rhein V, Song X, Wiesner A, Ittner LM, Baysang G, Meier F et al. Amyloid- $\beta$ and tau synergistically impair the oxidative phosphorylation system in triple transgenic Alzheimer's disease mice. Proc Natl Acad Sci USA 2009; 106: 20057-20062.

(c) (1) () $\odot$ This work is licensed under a Creative Commons Attribution-NonCommercial-NoDerivs 4.0 International License. The images or other third party material in this article are included in the article's Creative Commons license, unless indicated otherwise in the credit line; if the material is not included under the Creative Commons license, users will need to obtain permission from the license holder to reproduce the material. To view a copy of this license, visit http://creativecommons.org/licenses/by-nc-nd/4.0/ 